\title{
Monster Redshift Surveys through Dispersive Slitless Imaging: The Baryon Oscillation Probe
}

\author{
Karl Glazebrook, Ivan Baldry, Warren Moos, \\ Jeff Kruk, Stephan McCandliss \\ Dept. of Physics 8 Astronomy, Johns Hopkins Univ., Baltimore, MD 21218, USA
}

\begin{abstract}
Wide-field imaging from space should not forget the dispersive dimension. We consider the capability of space-based imaging with a slitless grism: because of the low near-infrared background in space and the high sky-density of high redshift emission line galaxies this makes for a very powerful redshift machine with no moving parts. A small $1 \mathrm{~m}$ space telescope with a 0.5 degree field of view could measure redshifts for $10^{7}$ galaxies at $0.5<z<2$ per year, this is a MIDEX class concept which we have dubbed 'The Baryon Oscillation Probe' as the primary science case would be constraining dark energy evolution via measurement of the baryonic oscillations in the galaxy power spectrum. These ideas are generalizable to other missions such as SNAP and DESTINY.
\end{abstract}

\section{Dispersive Space Imaging}

Slitless dispersive imaging has a long history extending back to the early Schmidt plate objective prism surveys. The set up is an imaging system, a dispersing element (ideally located at a pupil of the imaging system to avoid aberrations, but this is not essential in slow beams) and a filter to delimit the bandpass. In essence the object forms its own slit due to its angular size, to first order the background over the field of view is uniform and is equal to the undispersed background through the same filter. From the ground this means the background remains bright when the object's light is dispersed and so one takes an enormous signal-to-noise $(\mathrm{S} / \mathrm{N})$ penalty. Because of this such surveys have generally been restricted to bright emission line galaxies in the local Universe (see the KISS survey[1] for a recent example). For unresolved emission lines the $\mathrm{S} / \mathrm{N}$ is independent of the spectral resolution of the disperser. 
A low background environment greatly increases the sensitivity of slitless surveys. In space the background is dominated by solar scattered light from zodiacal dust and at 1.6 microns is $\sim 1000 \times$ darker than on the ground [2] (there is some dependence on ecliptic latitude). On the Hubble Space Telescope both the ACS optical camera and the NICMOS near-infrared camera are equipped with grisms for slitless spectroscopy. This mode has been used on ACS for spectroscopy of Type 1a supernovae to $z=2[3]$ and on NICMOS for detection of an abundant population of $z>1 \mathrm{H} \alpha$ emission line galaxies [4]. The strong evolution of the cosmological star-formation rate out to $z=1$ means that their are many more bright emission line galaxies than would otherwise be the case[5]. The space background peaks at $0.6 \mu \mathrm{m}$ and so as one follows $\mathrm{H} \alpha$ to high-redshift both the strong evolution in the source population and the diminishing background act to counteract the cosmological dimming for the S/N. Going from $z=0.2$ to $z=2$ these effects work to give us a S/N boost of a factor of 30 and make the high-redshift regime accessible to small space telescopes.

\section{The Baryon Oscillation Probe}

During discussions at Johns Hopkins in 2003-2004 we developed a strawman concept for a MIDEX class space mission which could do large scale slitless redshift surveys. We called this the 'Baryon Oscillation Probe' as our primary science motivation was to probe the baryonic oscillations in the power spectrum of galaxy clustering (see below). Our strawman concept consisted of a $1 \mathrm{~m}$ telescope with a $0.5^{\circ} \mathrm{FOV}$ operating in the $1-2 \mu \mathrm{m}$ near-infrared bandpass. This field would be sampled with 0.5 arcsec pixels giving a $4096 \times 4096$ detector array requirement. A set of low-dispersion fixed grisms and filters would provide for dispersed slitless imaging. Our rough order-of-magnitude estimates of size, mass and cost put this spacecraft in the MIDEX class. Initial studies show that BOP could be done either in LEO (with active cooling) or at L2 (passive cooling). We use this strawman to demonstrate the power of the slitless concept.

The exposure times depend on redshift, the response of the system and the choice of blocking filters. Cosmological dimming makes the highest redshifts more difficult. Our baseline goal was to measure $\mathrm{H} \alpha$ over the redshift range $0.5<z<2$, this is the redshift range optimal for testing dark energy models [7]. To determine exposure times we use published luminosity functions for $\mathrm{H} \alpha$ in this redshift range [5]:

$$
\left[\log \left(L^{*} / \mathrm{W}\right), \log \left(\phi^{*} / \mathrm{Mpc}^{-3}\right), \alpha\right]=[35.9,-3.1,-1.6]
$$


and set a luminosity limit as a function of redshift which gives critical number density sampling of the galaxy power spectrum for the purposes of the baryon oscillation science. We assumed the luminosity function $L^{*}$ evolved as $(1+z)^{3}$ over $0<z<1$ and was constant for $z>1$. This is a good match to the observed data of the cosmological star-formation rate evolution [6]. Following [8] we adopt a density of galaxies $n$ sufficient that the power spectrum $P(k)$ measurement is not shot-noise limited $(n P=3$ in their criteria) and also a bias model for galaxies which evolves with redshift in order to match observations of low and high-redshift galaxy clustering $\left(r_{0}=5 h^{-1} \mathrm{Mpc} \simeq\right.$ const.). This gives an $\mathrm{H} \alpha$ flux limit which is a function of redshift reaching $10^{-16} \mathrm{ergs} \mathrm{cm}^{-2}$ $\mathrm{s}^{-1}$ and a number density of $2000 \mathrm{deg}^{-2}$ for the $1.75<z<2$ bin. As a cross-check on our luminosity function calculation we can count directly the number of sources in redshift bins vs. flux in the NICMOS grism fields[5] and find agreement with our numbers.

Given these flux limits the main design parameter affecting exposure times is the combined throughput vs. wavelength of the grism and blocking filter. A wider filter lets through $\mathrm{H} \alpha$ over a wider redshift range but also lets in more background which reduces $\mathrm{S} / \mathrm{N}$. Because the background spectrum is not flat it is advantageous to have a set of narrow filters rather than one large filter. Further the background is higher at shorter wavelengths, i.e. low redshifts where the required flux limit is less deep. This turns out to be critical - if we have the freedom to have an abitrary response function we find that an optimized filter would deliberately have a declining throughput at blue wavelengths. This reduces the background, benefiting long-wavelength highredshift $\mathrm{H} \alpha$. The sensitivity is reduced at low redshift but the lines are brighter so it does not matter. Figure 1 shows a hypothetical two filter set designed in this manner which would give a uniform exposure time (1800 secs) for the whole $0.5<z<2$ range. We have not explored the feasibility of such filters, we note that to achieve the desired behavior one could change both the filter response or the grism response or a combination of both.

One issue for slitless surveys is ambiguous line identification. In principle multi-color photometry can be used to estimate redshifts and remove this ambiguity. However it is interesting to consider how well one can do with a single line. Using our evolving line luminosity function model we can estimate this by adopting reasonable values for fixed emission line ratios and assuming all the lines following the same cosmological evolution. We find that at our required flux limits $\mathrm{H} \alpha$ dominates, for example at $z=1.9$ it contributes $72 \%$ of the sources. The principal contaminant turns out to be [SIII] $9532 \AA$ which aliases $\mathrm{H} \alpha$ at $z=1.1$ (27\% of line sources). Even very crude broad-band data would suffice to eliminate such low-redshift galaxies. For [OIII] at $z=2.8$ and [OII] at $z=4.1$ we find contamination rates of $0.5 \%$ and $0.07 \%$ respectively their fluxes are heavily suppressed by cosmological dimming. These fractions were based on the assumption that $L^{*} / L_{\mathrm{H} \alpha}^{*}=(0.2,0.3,0.5)$ for [SIII], [OIII] 


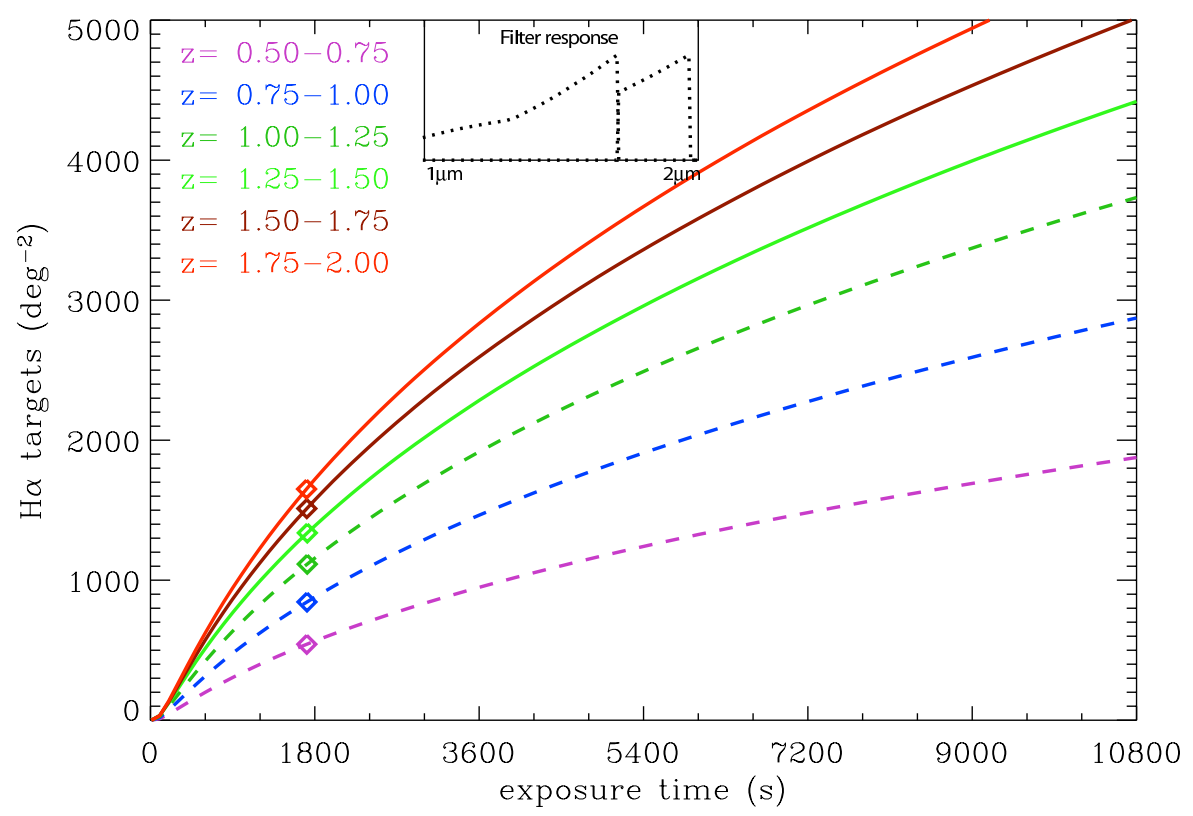

Fig. 1. Predicted number density of targets seen from BOP vs. exposure times for different redshift ranges using an optimized two filter set. The diamonds show the required number density for the baryon oscillation science at each redshift. The filters have been optimized to give equal exposure times for these points. The throughputs ( + grism response) of the optimized filter set vs. wavelength are shown in the inset. We assume a peak efficiency of $38 \%$ (effective area of $0.3 \mathrm{~m}^{2}$ ) for first-order diffraction and that other orders do not contribute significantly to the background. and $[\mathrm{OII}]$, respectively.

We also considered an alternate approach to BOP using the ultraviolet rather than the near-infrared and targeting Ly $\alpha$ emitters. We constructed an empirical model of their line luminosity function which matches the observed UV continuum luminosity function and Ly $\alpha$ equivalent width distribution of $z \sim 3$ Lyman break galaxies $[9,10]$. We found the UV approach was not competitive - the exposure times were an order of magnitude larger. This is a combination of the much lower strength of the Ly $\alpha$ line and the fact that the background now increases towards higher redshifts $(0-3)$.

\section{Baryon Oscillations and Dark Energy constraints}

Of course there many possible science experiments BOP could address (see Conclusions). We choose to focus on one for illustration of the redshift survey power: detection of the baryon oscillations in the galaxy power spectrum and 
resulting constraints on dark energy.

Based on the field of view and exposure time we calculate BOP can redshift survey $2000 \mathrm{deg}^{2}$ and $10^{7}$ objects per year. We assume our two filter optimized design and that each field is observed at two roll angles to improve deconvolution of spectral overlap. (This is also desirable to allow the zero point of the wavelength scale of each object to be determined, alternatively zeroth order could be used.)

The spectral resolution requirement remains to be determined precisely. Blake \& Glazebrook [8] determined for full $P(k)$ resolution: $\sigma_{z} /(1+z) \simeq 0.001$, which translates to $R=125$ if the line can be centroided to a quarter of a resolution element at $S / N=10$. However to resolve the nearby $\mathrm{H} \alpha$ and [NII] lines requires $R>400$ so this might be preferable.

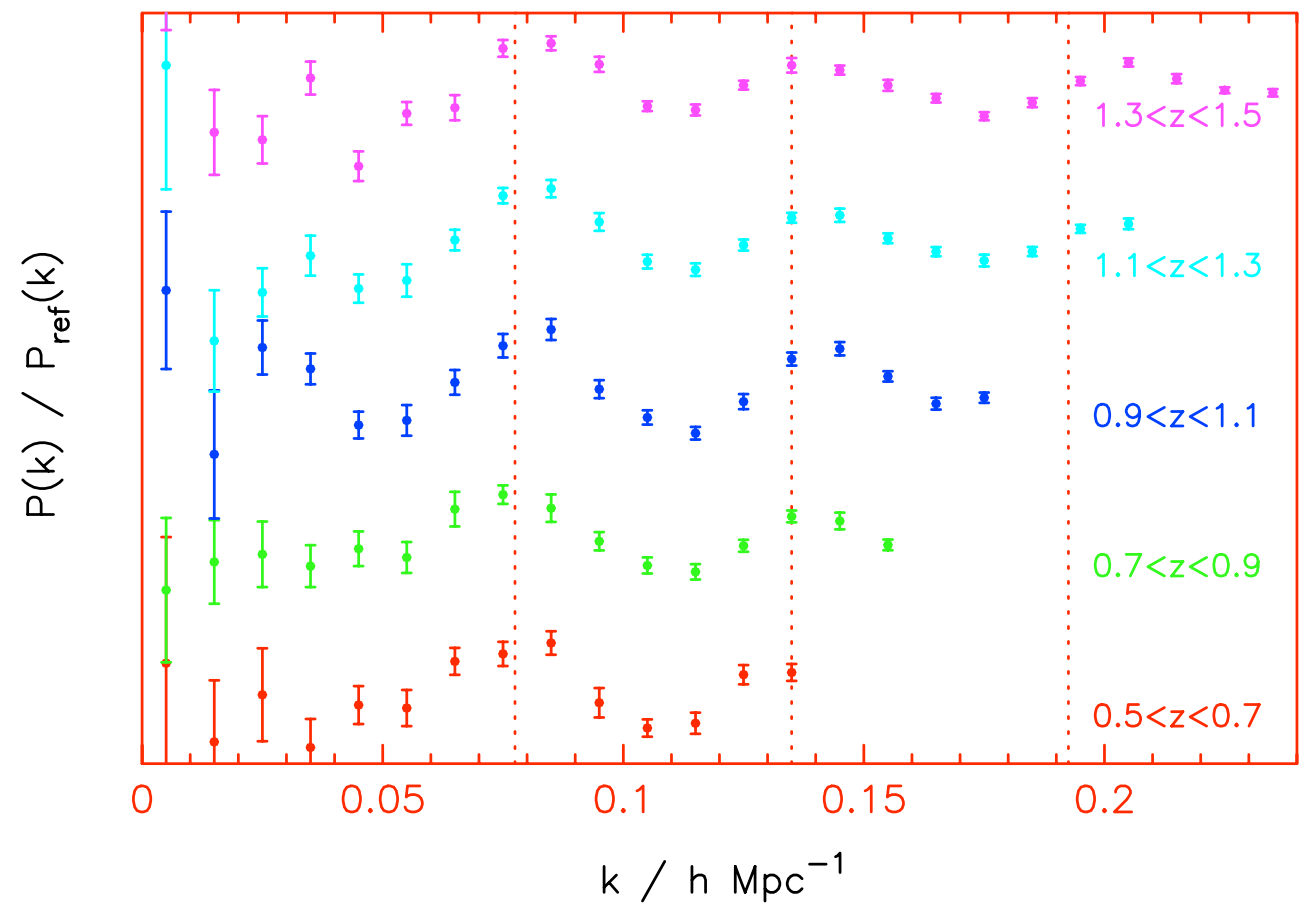

Fig. 2. Predicted galaxy power spectrum for a $10,000 \mathrm{deg}^{2}$ survey. The non-oscillatory component has been divided out and the power spectrum (linear regime only) is shown in redshift bins. The small error bars show the precise measurement possible on the baryonic oscillations (the 'standard ruler' for the dark energy test) possible with a survey of this size. In this case the model data is for a $w=-0.9$ simulated cosmology, the peaks visibly shift from their location in a $w=-1$ cosmology (vertical dotted lines).

We assume a 5 year mission resulting in a survey of $10,000 \mathrm{deg}^{2}$ and apply the methodology of Glazebrook \& Blake (2004; in prep.) to simulate the recovered power spectrum (Figure 2) and resulting dark energy constraints (Figure 3). The constraints are comparable to those delivered using the supernova technique by the Supernova Acceleration Probe (SNAP [11]). As with the 


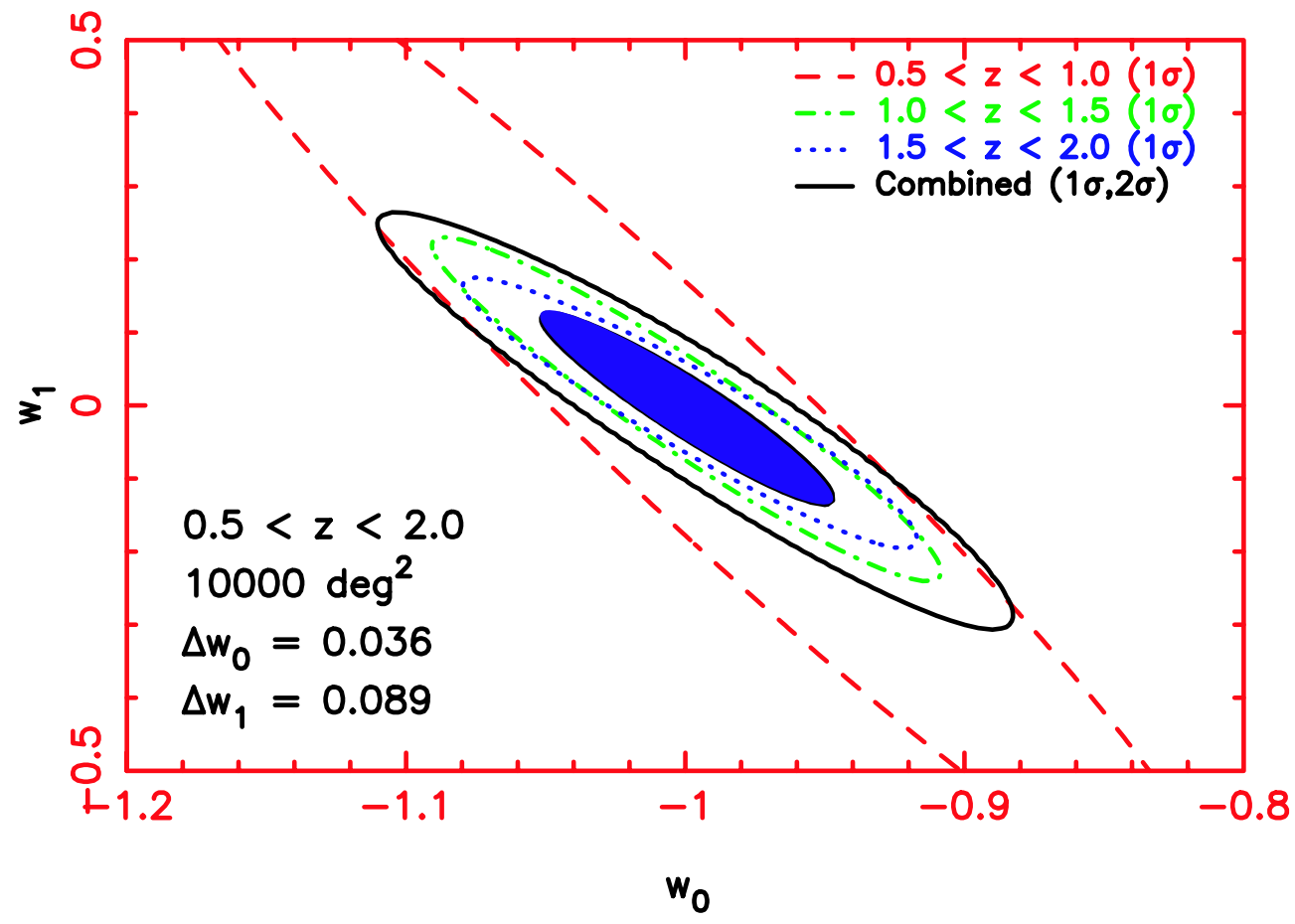

Fig. 3. Recovered constraints on a dark energy equation of state $w(z)=w_{0}+w_{1} z$ from a $10,000 \mathrm{deg}^{2} \mathrm{BOP}$ survey. It can be seen that each redshift range is slightly non-degenerate with the others, the combined constraints are very tight and are comparable to the precision delivered using supernovae in the SNAP mission.

supernovae this requires independent accurate constraints on other cosmological parameters (e.g. $\Omega_{m}, H_{0}$ ) from local surveys and the Cosmic Microwave Background measurements — we refer the reader to Glazebrook \& Blake for a detailed discussion.

We note this proposed redshift survey improves by a factor of ten on the most ambitious proposed future ground-based redshift survey. This is the KAOS concept [13] for the Gemini Telescope which could survey $1000 \operatorname{deg}^{2}$.

It is also interesting to consider that there is very little conceptual difference between BOP and any wide field imaging space mission. Both require large detector arrays and wide field optics, the only difference is provision of a grism. For example one could merge BOP with SNAP — this would add a completely independent method to the dark energy determination and with similar precision. It would also mean that the many millions of high-redshift galaxies imaged with SNAP could get spectroscopic follow-up in the same mission. Alternately BOP is very similar to the DESTINY concept [12] which uses slitless spectroscopy to locate high-redshift supernovae (albeit at somewhat lower resolution). 


\section{Conclusions}

We have outlined a concept (BOP) for a near-infrared space mission using slitless spectroscopy to do wide area redshift surveys an order of magnitude larger than existing ground based surveys such as SDSS and 2dFGRS in both number and volume and probing high-redshift $(0.5<z<2)$. It represents a factor of ten improvement on what could be done on the ground. Instrumentally it represents a very simple hardware system which by taking advantage of the low background in space has no requirement for complex fiber or slit configuring machinery. Although lower backgrounds could be achieved with wide field spectroscopic fiber or slit designs that have recently been proposed[14] these technologies are immature by spaceflight standards and as such must be considered risky at the present time.

BOP would serve diverse science goals: some other examples include studying the evolution of galaxy clustering, measuring the evolving luminosity function

of star-forming galaxies as a function of metallicity and environment, providing redshift depth information for deep wide imaging surveys and searching for Ly $\alpha$ emission from young galaxies at $z>7$. We have presented in detail BOP's ability to constrain dark energy using baryon oscillation measurements. It would deliver precision on the equation of state comparable to the SNAP mission but via a completely independent technique.

The BOP concept is generalizable to any wide-field space mission as long as a grism could be inserted in to the imaging system. We argue that in any such space mission the science-to-cost ratio of adding such a grism is enormous, and should be considered.

\section{Acknowledgements}

We would like to thank Chris Blake for assistance in producing Figures 2 and 3 and Bob Woodruff from Lockheed Martin for useful design discussions. KG acknowledges generous funding from the David and Lucille Packard foundation.

\section{References}

[1] Wegner, G., Salzer, J. J., Jangren, A., Gronwall, C., \& Melbourne, J. 2003, AJ, 125,2373 
[2] The JWST Background Simulator. http://www.stsci.edu/jwst/science/jms/jms_bgnd_form.html

[3] Riess, A. G., et al. 2004, ApJ, 607, 665

[4] McCarthy, P. J., et al. 1999, ApJ, 520, 548

[5] Hopkins, A. M., Connolly, A. J., \& Szalay, A. S. 2000, AJ, 120, 2843

[6] Glazebrook, K., et al. 2003, ApJ, 587, 55

[7] Linder, E. V. \& Huterer, D. 2003, Phys. Rev. D, 67, 081303

[8] Blake, C. \& Glazebrook, K. 2003, ApJ, 594, 665

[9] Steidel, C. C., Adelberger, K. L., Giavalisco, M., Dickinson, M., \& Pettini, M. 1999, ApJ, 519, 1

[10] Shapley, A. E., Steidel, C. C., Pettini, M., \& Adelberger, K. L. 2003, ApJ, 588, 65

[11] Aldering, S. C. G., et al. 2004, PASP in press, astro-ph/0405232

[12] Lauer, T. R., et al. 2004, this proceedings

[13] Dey, A., et al. 2003, The KAOS Purple Book

[14] McCandliss, S. R., et al. 2004, SPIE 5488, in press, (astro-ph/0407464) 